\title{
Effet des touffes de Guiera senegalensis (J.F. Gmel) sur la fertilité des sols dans la région de maradi (Niger)
}

\author{
DAN LAMSO Nomaou, GUÉRO Yadji, TANKARI DAN-BADJO Abdourahamane*, TIDJANI Adamou \\ Didier, ADO MAMAN Nassirou, AMBOUTA JEAN MARIE Karimou \\ Département Sciences du sol, Faculté d'Agronomie de Niamey \\ Université Abdou Moumouni de Niamey, Niamey, Niger \\ E-mail: tankari@yahoo.fr
}

Original submitted in on 25th August 2015. Published online at www.m.elewa.org on 31st October 2015

http://dx.doi.org/10.4314/jab.v94i1.8

\section{RÉSUMÉ}

Objectifs : A Guidan Bakoye, dans la région de Maradi au Niger, pour faire face à la dégradation et la baisse de la fertilité des terres, les paysans ont entre autres adopté la régénération naturelle assistée des espèces ligneuses telles que Guiera senegalensis (Guiera du Sénégal). Cette étude traite de l'influence des touffes de cet arbuste sur la fertilité des sols dans le terroir de Guidan Bakoye.

Méthodologies et résultats : Un dispositif en blocs randomisés complet avec trois répétitions des touffes, a été utilisé en plein champ. Des prélèvements des sols ont été effectués autour des touffes selon les points cardinaux, les distances $0,50,100,150$ et $200 \mathrm{~cm}$ de la touffe et la profondeur du sol $(0-10 ; 10-20,20$ 30 et $30-40 \mathrm{~cm}$ ), et leurs caractéristiques physico-chimiques ont été déterminées. Les résultats obtenus ont révélé généralement une amélioration significative des paramètres physico-chimiques des sols localisés à proximité des touffes de Guiera senegalensis.

Conclusion et application de la recherche: II ressort de cette étude, un fort taux de repeuplement de Guiera senegalensis (1800 pieds/ha) dans le terroir grâce à la régénération naturelle assistée et à l'introduction de la technique de marcottage. Par ailleurs, les résultats des analyses physico-chimiques des sols sous Guiera senegalensis, ont montré l'absence de changement textural avec la distance, et la prédominance des sables dans les horizons de surface indiquant que les touffes de Guiera senegalensis piègent essentiellement les sables transportés par les vents. II est aussi apparu que les touffes de Guiera senegalensis ne semblent pas influencer le $\mathrm{pH}$, la capacité d'échanges cationiques et le $\mathrm{Ca}$ contrairement à la matière organique, au phosphore, $\mathrm{Mg}$ et $\mathrm{K}$. En définitive, les touffes de Guiera senegalensis améliorent moyennement les propriétés physico-chimiques du sol.

Mots clés : Touffes, Guiera senegalensis, paramètres physico-chimiques des sols, Guidan Bakoye, Maradi 


\section{Nomaou et al. J. Appl. Biosci. 2015 Effet des touffes de Guiera senegalensis (J.F. Gmel) sur la fertilité des sols dans la région de Maradi (Niger)}

\section{Effect of Guiera senegalensis (JF Gmel) tufts on soil fertility in Maradi (Niger) \\ ABSTRACT}

Objectives: In arid and semi-arid, the degradation and the decline in the fertility of land is a major concern for farmers. At Guidan Bakoye, in the Maradi region, to cope with this, the farmers have adopted the assisted natural regeneration to rebuild a degraded environment due to uncontrolled use. Thus, woody species like Guiera senegalensis (Guiera du Sénégal) have been bred and maintained in this region. The present study deals with the influence of the tufts of this plant on the soil fertility of Guidan Bakoye.

Methodology and Results: A device block randomized complete with three repetitions of the tufts, was used in the field. Soil Samples were carried out around the tufts according to the cardinal points, the distances of $0,50,100,150$ and $200 \mathrm{~cm}$ of the tuft and the soil depth $(0-10 ; 10-20,20-30$ and $30-40 \mathrm{~cm})$.

Conclusion and application of research: This study show a high rate of Guiera senegalensis repopulation (1800 plants/ha) in the soil through assisted natural regeneration and the introduction of the technique of layering. In addition, the results of the physico-chemical analysis of soils under Guiera senegalensis have shown the absence of textural change with the distance, and the predominance of sand in the surface horizons, indicating that the tufts of Guiera senegalensis trap essentially the sand transported by the wind. It is also apparent that the tufts of Guiera senegalensis did not seem to influence the $\mathrm{pH}$, the capacity of the cation exchange and the $\mathrm{Ca}$ in contrast to the organic matter, phosphorus, $\mathrm{Mg}$ and $\mathrm{K}$. In the end, the tufts of Guiera senegalensis improve moderately physico-chemical properties of the soil.

Keywords : Tufts, Guiera senegalensis, physico-chemical parameters of the soil, Guidan Bakoye, Maradi

\section{INTRODUCTION}

Au Niger et particulièrement dans la région de Maradi, l'agriculture est la principale activité économique. Mais, cette dernière rencontre d'énormes contraintes liées à la saturation de l'espace cultivable, la mauvaise répartition de la pluviométrie dans le temps et dans l'espace, la forte pression démographique et la baisse de la fertilité des sols. Cela engendre de nombreuses conséquences dont l'insuffisance de la production agricole, et par suite une insécurité alimentaire devenue quasi-chronique et plus sévère en milieu rural qu'en milieu urbain (Strebelle et Boubacar, 2011). Face à cette situation, plusieurs stratégies ont été adaptées ou développées par les paysans. Parmi ces stratégies, on peut citer les techniques classiques de conservation des eaux et des sols et de défense et restauration des sols, la rotation culturale, l'association céréales - légumineuses et l'utilisation des espèces agroforestières dans les champs (Larwanou et al., 2006 ; Bationo et al., 2012). Dans le département d'Aguié, en particulier dans les villages de Dan Saga et Guidan Bakoye, les paysans ont protégé et entretenu les arbres qui poussent dans leurs champs pendant le défrichement. Grâce à cette pratique appelée
Régénération Naturelle Assistée (RNA), la densité des ligneux dans les champs s'est améliorée, avec environ 60 à 100 individus à I'hectare (Salissou, 2004 ; Hamissou, 2005 ; Moustapha, 2005). Des travaux de recherche ont traité de l'utilisation des espèces ligneuses dans les champs telles que Acacia albida (Maï Moussa, 1996 ; Maï Moussa et al., 1997), Hyphaene thebaica (Moussa, 1997, Dan Lamso et al., 2015), Boracus aethiopum (Kadadé, 1999) et Acacia senegal (Abdou et al., 2013) dans l'optique d'améliorer la fertilité des sols et les productions agricoles. En vue d'une meilleure compréhension des interactions entre arbustes et sols et afin d'améliorer la durabilité du système, la présente étude est conduite sur l'effet des touffes de Guiera senegalensis sur la fertilité des sols dans le terroir de Guidan Bakoye dans le département d'Aguié. II s'agit d'une part, de déterminer la place de l'arbuste Guiera senegalensis dans ce terroir et d'autre part, d'étudier son influence sur certains paramètres physico-chimiques des sols (texture, $\mathrm{pH}$, matière organique, phosphore, bases échangeables, capacité d'échange cationique). 


\section{Nomaou et al. J. Appl. Biosci. 2015 Effet des touffes de Guiera senegalensis (J.F. Gmel) sur la fertilité des sols dans la région de Maradi (Niger)}

\section{MATERIEL ET METHODES}

Présentation et choix du site d'étude : L'étude s'est déroulée dans le terroir villageois de Guidan Bakoye situé à $22 \mathrm{~km}$ au Nord d'Aguié entre 13\%41'35" de latitude nord et $07^{\circ} 46^{\prime} 483^{\prime \prime}$ de longitude Est comme le montre la figure 1. Le terroir est limité à l'Est par Woura, à l'Ouest par Dan Saga, au Sud par Guidan Dawey et au Nord par Dogarawa.

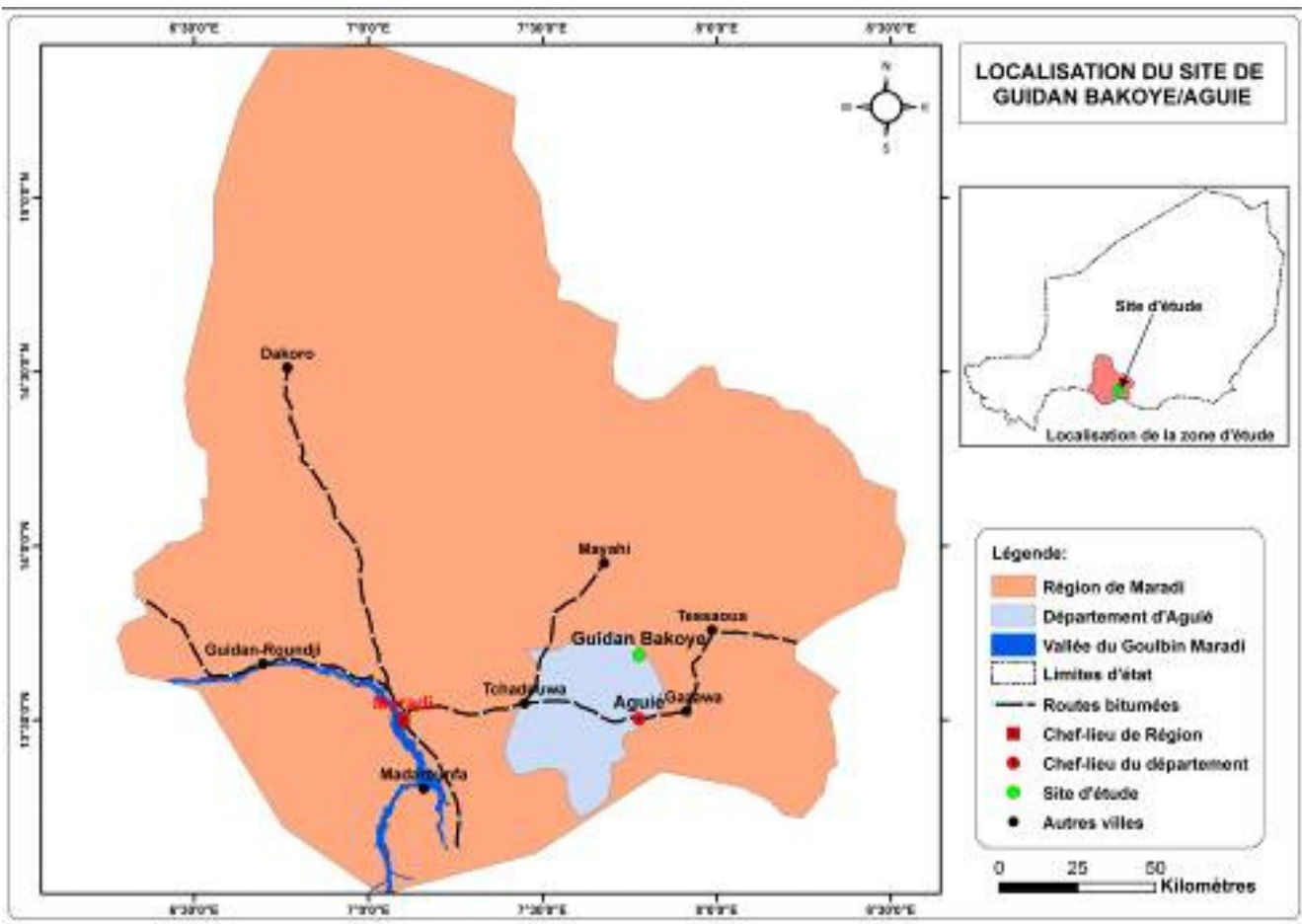

Figure 1 : Localisation du site de Guidan Bakoye

Le climat de Guidan bakoye est de type sahélien avec une pluviométrique annuelle moyenne comprise entre 400 et $600 \mathrm{~mm}$. L'agriculture pluviale constitue ainsi la principale activité socio-économique des populations. Les cultures dominantes sont le mil, le sorgho, le niébé et l'arachide et secondairement le sésame, le voandzou et l'oseille. Cependant, cette activité connait plusieurs contraintes notamment la baisse de la fertilité des sols, une crise foncière agricole, l'insuffisance et l'irrégularité des pluies et des pressions parasitaires. On note aussi une très faible mécanisation de l'agriculture et un faible accès aux autres intrants agricoles. Les sols du terroir sont dominés essentiellement par les sols ferrugineux tropicaux développés sur du sable et appelés " Jigawa » (Ambouta et al., 1998). Le couvert végétal du terroir est marqué par la prédominance des Combrétacées notamment Guiera senegalensis qui constitue non seulement la principale ressource fourragère mais aussi la première source de bois de chauffe. Le choix du terroir de Guidan Bakoye a été fait selon les critères suivants: densité de Guiera senegalensis, parcelles expérimentales non loin $\mathrm{du}$ village et devant représenter le système de culture dominant du terroir. A cet effet, un champ présentant une densité de 1800 touffes/ ha de Guiera senegalensis a été choisi.

Matériel végétal : Guiera senegalensis est une espèce de la famille des Combretaceae, du genre Guiera, espèce senegalensis. C'est un arbuste d'environ 3 mètres de haut se présentant quelquefois sous l'aspect de buissons ne dépassant pas 1,5 $\mathrm{m}$ (Arbonnier, 2009) comme le montre la figure 2. II présente de petites feuilles persistantes et opposées qui sont vertes plus ou moins grises ou bleutées, ovales - arrondies, un peu duveteuses sur les deux faces (Arbonnier, 2009). Ses fleurs, blanches ou jaunâtres, à pétales fort étroits, constituent de denses têtes pédonculées et ses fruits, droits, sont lobés en "patte d'araignée", densément couverts de très longs poils soyeux. Le fruit est un akène linéaire brun à vert de 3 à $4 \mathrm{~cm}$ à pubescence 


\section{Nomaou et al. J. Appl. Biosci. 2015 Effet des touffes de Guiera senegalensis (J.F. Gmel) sur la fertilité des sols dans la région de Maradi (Niger)}

argentée. Son écorce est lisse ou écailleuse et de couleur grise (Arbonnier, 2009). Les racines de Guiera senegalensis sont vermifuges et à l'âge de 12 ans, Guiera senegalensis est à mesure d'envoyer latéralement ses racines jusqu'à $7,6 \mathrm{~m}$ du collet
(Loupe, 1991 et Bationo, 1994). C'est une espèce des zones soudano sahéliennes qui se développe bien sur les sols pauvres, sableux et cuirassés (Arbonnier, 2009 ; Thiombiano et al. 2006).

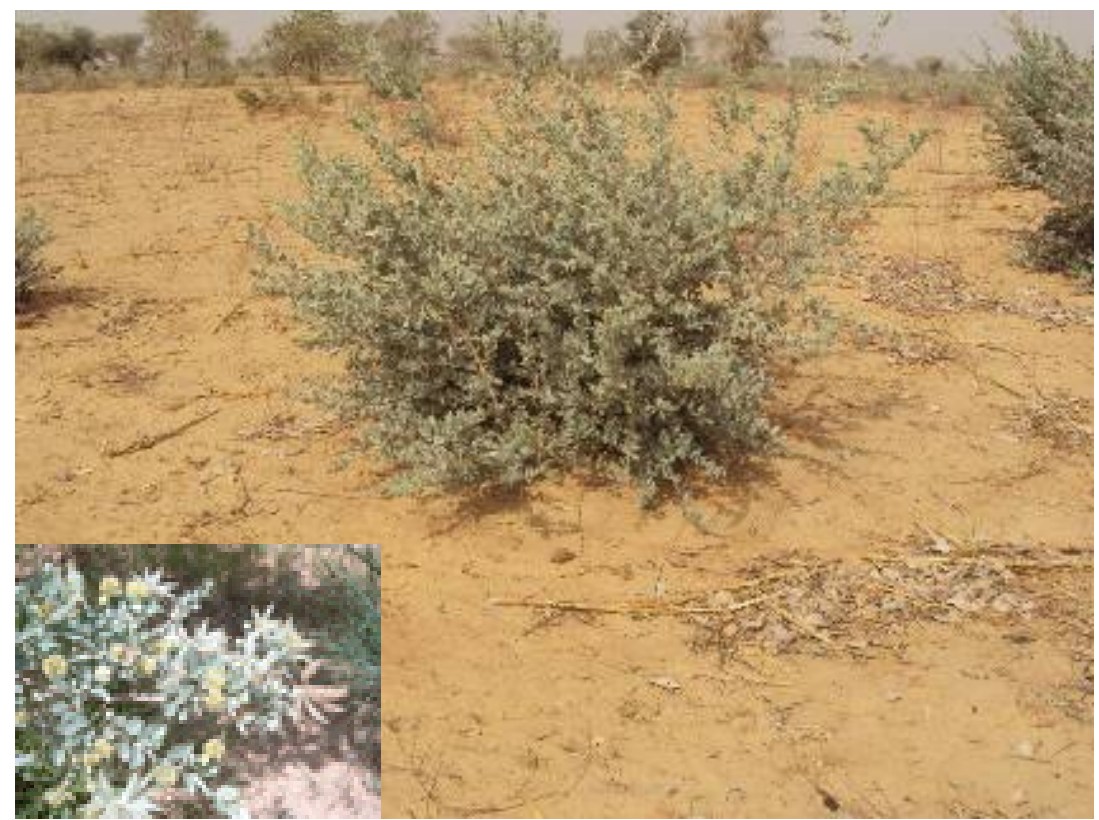

Figure 2 : Guiera senegalensis buissonnant

Enquête : II s'agit d'une enquête individuelle réalisée à base d'un questionnaire semi-ouvert adressé aux paysans. Les questions sont relatives au rôle et à l'importance écologique et socio-économique de palmier doum, son mode de gestion (RNA), paillis issu $\mathrm{du}$ recepage....) ainsi que la dynamique du peuplement. Ainsi, 15 paysans ont été questionnés dont 5 paysans les plus âgés du village, 5 paysans artisans qui utilisent les palmes et 5 paysans ayant de nombreux plants de RNA de Guiera senegalensis dans leurs champs. Les paysans ont été rencontrés dans leur champ ou au village.
Dispositif expérimental : II s'agit d'un dispositif en blocs randomisés complet avec trois répétitions des touffes de Guiera senegalensis. Le choix de la touffe (belou) s'est porté sur son isolement par rapport à une autre touffe et/ou un autre ligneux. Ainsi, les trois (3) touffes choisies sont distantes l'une de l'autre d'au moins $50 \mathrm{~m}$. Pour prélever les échantillons au niveau de touffe, on a déterminé d'abord la limite de la touffe selon les 4 côtés (Est, Ouest, Nord et Sud) ; ensuite l'échantillonnage s'est effectué latéralement tous les 50 $\mathrm{cm}$ à partir de la limite de la touffe jusqu'à une distance de $2 \mathrm{~m}$ conformément au dispositif de la figure 3 . 


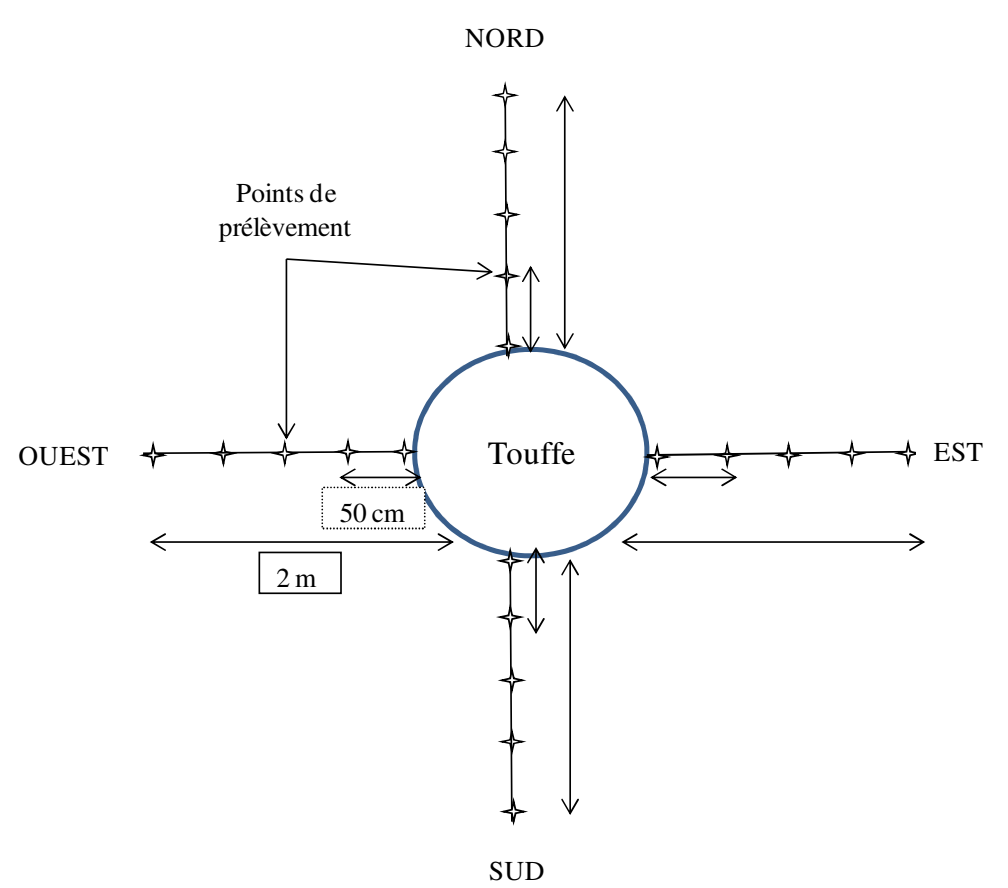

Figure 3 : Dispositif de prélèvement de sol autour de la touffe de Guiera senegalensis

Comme on peut le remarquer, chaque côté de la touffe porte 5 points de prélèvements et l'échantillonnage s'est effectué sur 4 profondeurs : $0-10 \mathrm{~cm}, 10-20 \mathrm{~cm}$, 20-30 $\mathrm{cm}$ et 30-40 $\mathrm{cm}$ par point. Ainsi il a été prélevé 20 échantillons des sols par côté soit 80 échantillons par touffe. Avec les trois répétitions des touffes, 240 échantillons sols ont été prélevés. Ces échantillons de sols ont fait l'objet d'analyses physico-chimiques au laboratoire du département science du sol de la Faculté d'Agronomie de I'Université Abdou Moumouni de Niamey.

Analyses physico-chimiques: Après séchage des échantillons à l'air ambiant du laboratoire, de leur broyage et de leur tamisage à $2 \mathrm{~mm}$, les méthodes résumées dans le tableau 1 ont été utilisées (Baize, 1988).

Tableau 1 : Méthodes d'analyses des sols utilisées

\begin{tabular}{c|c}
\hline PARAMETRES & METHODES \\
\hline $\mathrm{pH}$ & $\mathrm{pH}$-mètre avec rapport sol/eau 1/2,5 \\
\hline Granulométrie (6fractions) & $\begin{array}{c}\text { Pipette Robinson/tamisage après oxydation de la matière organique avec l'eau } \\
\text { oxygénée }\end{array}$ \\
\hline Carbone organique & Méthode Walkley \& Black (1934) \\
\hline Azote total & Kjeldahl \\
\hline Phosphore & Bray l \\
\hline CEC et bases échangeables & Méthode d'acétate d'ammonium pH $=7$ \\
\hline
\end{tabular}

Les différents modes opératoires sont sous forme de recueil au laboratoire de pédologie de la faculté d'Agronomie de l'université Abdou Moumouni de Niamey.

Analyse statistique des données: Les données d'analyses physico-chimiques des échantillons du sol ont été traitées avec le logiciel GenSTAT. Ainsi les données obtenues ont été soumises à une analyse de variance pour identifier la présence ou non de différences significatives (au seuil de 5\% de probabilité) pour les différents paramètres physico-chimiques selon la distance à la touffe, les points cardinaux, et la profondeur du sol. En présence de différences significatives, le test de Student Newman Keuls (au seuil de $5 \%$ ) a été effectué pour identifier les traitements significativement différents selon la variable considérée. 
RÉSULTATS ET DISCUSSION

\section{Guiera senegalensis dans le terroir de Guidan Bakoye \\ Densité de Guiera senegalensis: Le terroir de} Guidan Bakoye présente un important peuplement de Guiera senegalensis. Le recensement des touffes de Guiera senegalensis dans le champ où les essais ont été menés, donne une densité de 1800 pieds à l'hectare. Cette densité est nettement supérieure à celles observées au Mali et au Burkina Faso (800 pieds à l'hectare) par Bationo et al. (2012). Dans la partie Sud de Guidan Bakoye, cette densité est encore plus élevée. Dans les années 1970, le Nord de la région de Maradi qui inclut le terroir d'étude, a connu une grave crise écologie marquée par un fort recul des différentes combrétacées dû principalement à l'utilisation incontrôlée de ces ligneux comme bois d'énergie et fourrages et à l'extension des champs cultivés. Mais la tendance actuelle est au repeuplement grâce à la technique de la régénération naturelle assistée (Adam et al., 2006).

Rôles de Guiera senegalensis: Dans le terroir de Guidan Bakoye, les touffes de Guiera senegalensis subissent dans l'année 2 à 3 coupes au cours desquelles 1 à 2 tiges de rejets sont laissées et entretenues (figure 4). Au bout de 4 ans, ces rejets vont se développer et produire du bois au profit des ménages.

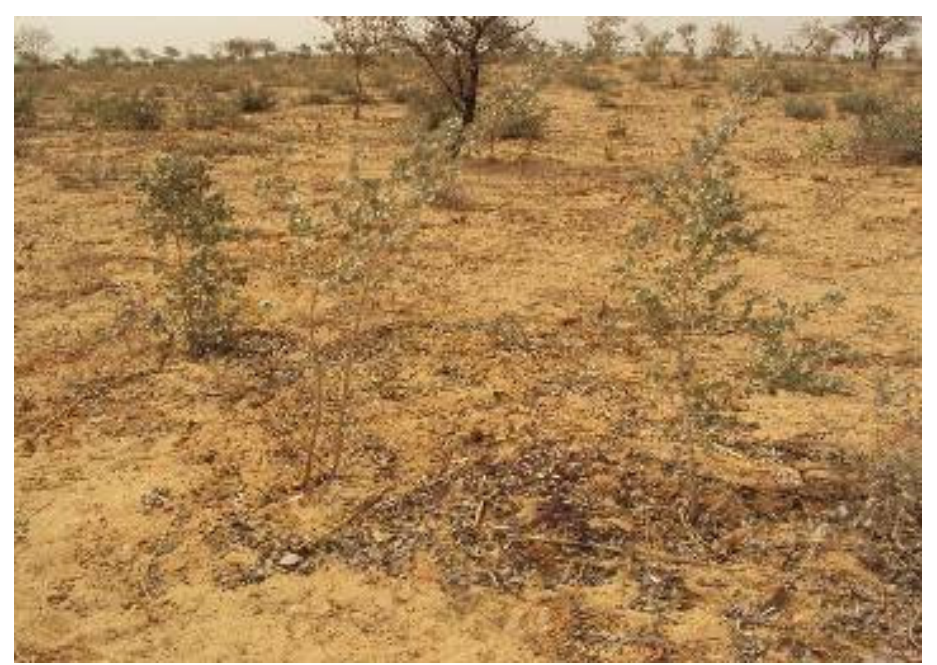

Figure 4 : Quelques tiges de Guiera senegalensis laissées après coupe des touffes

Les biomasses issues des coupes et d'élagage, sont laissées au sol non loin des souches ou mises en tas sur les parties du champ infertiles. Afin de préserver la ressource, les villageois ont mis en place un comité de surveillance qui travaille en rapport avec ceux des autres villageois pour prévenir les coupes frauduleuses. Aussi, compte tenu de la forte capacité de régénération de Guiera senegalensis, la technique de marcottage terrestre et aérien (Bationo et al., 2005) est introduite dans ce terroir. Ainsi, 271 marcottes ont été effectuées. Les premiers résultats ont montré une rapide émission des racines par la tige marcottée dès les deux premières semaines (figure 5) avec un taux de réussite de $90 \%$. 


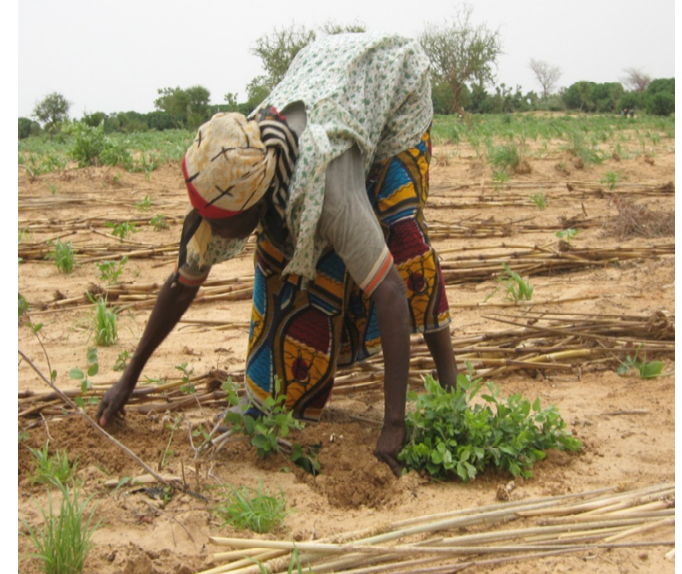

(a)

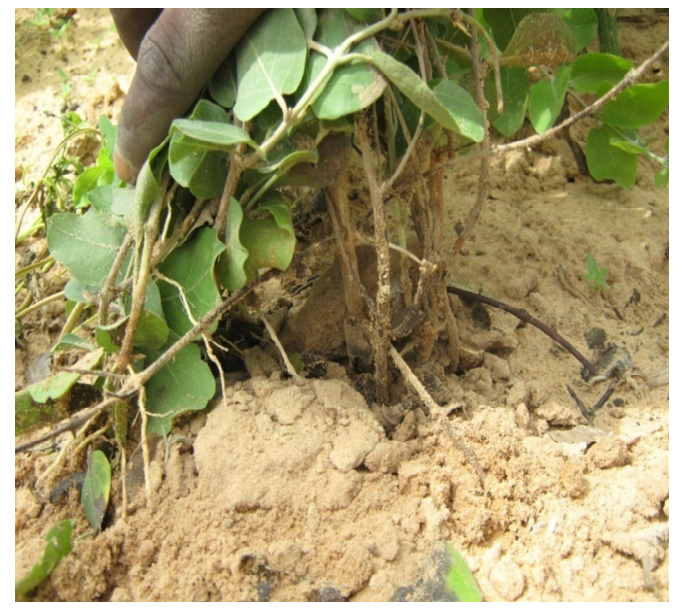

(c)

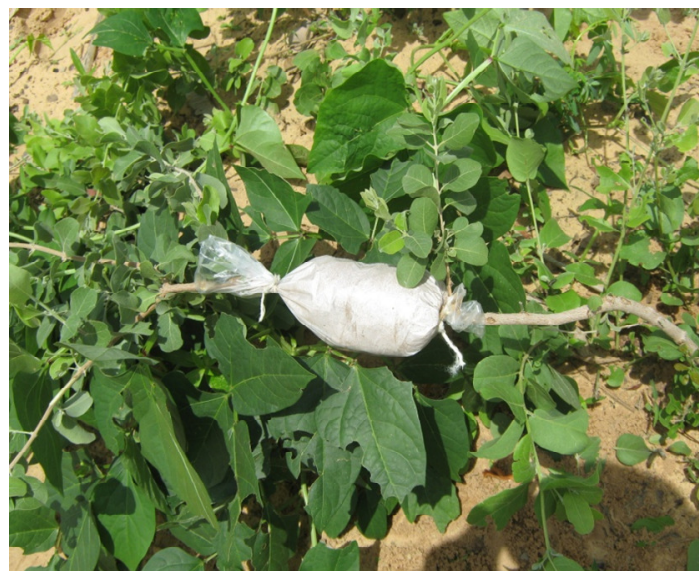

(b)

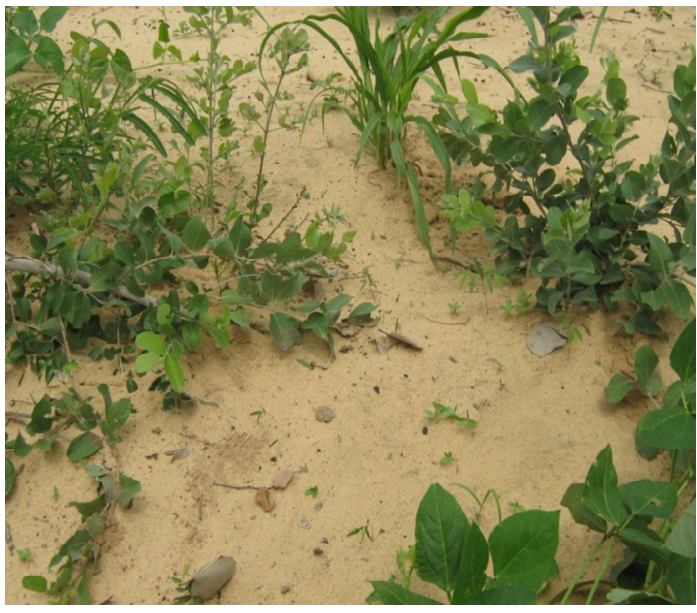

(d)

Figure 5 : Techniques de marcottage Guiera senegalensis à Guidan Bakoye :
(a) : Marcottage souterrain en cours ;
(b) : Marcottage aérien en place ;
(c) : émission des racines d'une marcotte ;
(d) : Marcotte en développement

En plus de leur contribution à la fourniture de la matière organique après leurs coupes, les touffes de Guiera senegalensis, retiennent par leur port buissonnant les feuilles des autres espèces transportées par les vents (figure 6). 


\section{Nomaou et al. J. Appl. Biosci. 2015 Effet des touffes de Guiera senegalensis (J.F. Gmel) sur la fertilité des sols dans la région de Maradi (Niger)}

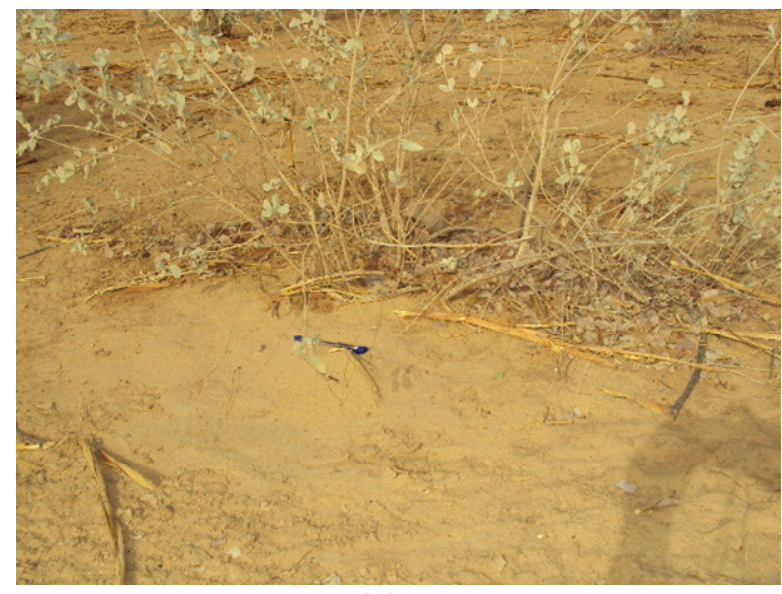

(a)

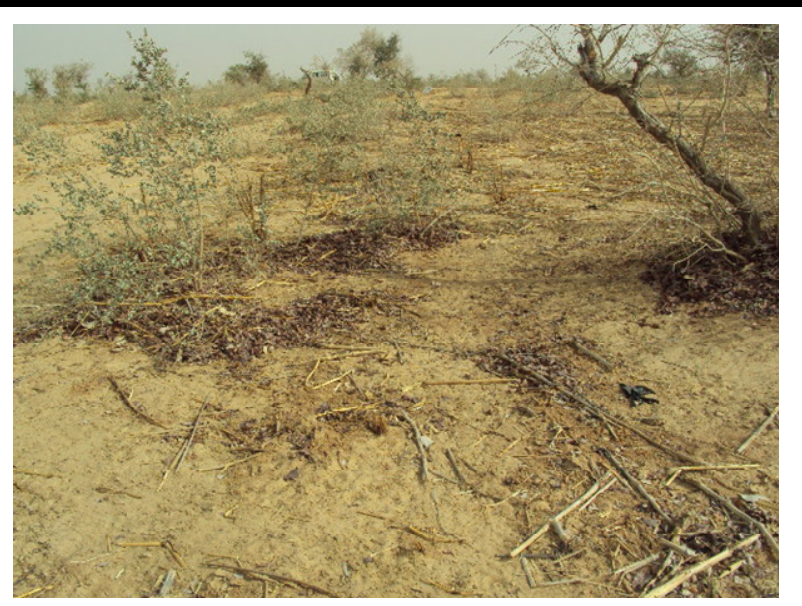

(b)

Figure 6 : Déflation (a) et piégeage des substrats organiques (b) transportés par les vents autour des touffes de Guiera senegalensis

Ce rôle de piégeage des débris par les touffes de Guiera senegalensis est moindre lorsque leur port est érigé. Aussi, la déflation constatée au pied de cette espèce (figure 6a), semble contredire de nombreux travaux qui ont mis en évidence la formation autour des touffes de Guiera senegalensis, d'une microbutte de plus de $15 \mathrm{~cm}$ de haut et d'1 $\mathrm{m}$ de large (Louppe, 1991 ; Bationo, 1994 ; Wezel et al., 2000). Enfin, les racines, les rameaux et les feuilles de Guiera senegalensis sont également utilisés dans la pharmacopée traditionnelle pour traiter notamment les plaies, la diarrhée et la dysenterie.

Variation des paramètres physico - chimiques du sol autour des touffes de Guiera senegalensis Distribution granulométrique: Le tableau 2 donne les résultats d'analyse de variance des différentes fractions granulométriques des sols prélevés autour des touffes de Guiera senegalensis.

Tableau 2 : Analyse de variance des différentes fractions granulométriques des sols

\begin{tabular}{|c|c|c|c|c|c|c|}
\hline \multirow[t]{2}{*}{ Source de variance } & \multicolumn{5}{|c|}{ Les moyennes selon les distances } & \multirow[t]{2}{*}{ Fpr (5\%) } \\
\hline & $0 \mathrm{~cm}$ & $50 \mathrm{~cm}$ & $100 \mathrm{~cm}$ & $150 \mathrm{~cm}$ & $200 \mathrm{~cm}$ & \\
\hline Argile & $1,41^{\mathrm{a}}$ & $1,3^{\mathrm{a}}$ & $1,20^{\mathrm{a}}$ & $1,28^{a}$ & $1,22^{\mathrm{a}}$ & 0,602 \\
\hline Limon & $5,37^{a}$ & $5,11^{a}$ & $5,27^{a}$ & $5,37^{a}$ & $5,24^{a}$ & 0,3796 \\
\hline Sable & $93,52^{\mathrm{a}}$ & $93,55^{\mathrm{a}}$ & $93,45^{a}$ & $93,30^{a}$ & $93,39 a$ & 0,914 \\
\hline \multicolumn{7}{|c|}{ Les moyennes selon les directions } \\
\hline & $E$ & & & & $\mathbf{S}$ & Fpr $(5 \%)$ \\
\hline Argile & \multicolumn{2}{|l|}{$1,57^{b}$} & $1,22^{\mathrm{a}}$ & $8^{\mathrm{a}}$ & $1,17^{a}$ & 0,004 \\
\hline Limon & \multicolumn{2}{|l|}{$5,22^{b}$} & $4,91^{a}$ & $99 \mathrm{c}$ & $4,97^{a}$ & $<0,001$ \\
\hline Sable & $93,75^{b}$ & & & & $93,86^{\mathrm{ab}}$ & $<0,001$ \\
\hline \multicolumn{7}{|c|}{ Les moyennes selon les profondeurs } \\
\hline & \multicolumn{2}{|l|}{$10 \mathrm{~cm}$} & $20 \mathrm{~cm}$ & $30 \mathrm{~cm}$ & $40 \mathrm{~cm}$ & Fpr (5\%) \\
\hline Argile & \multicolumn{2}{|l|}{$1,20^{\mathrm{a}}$} & $1,33^{a}$ & $1,15^{\mathrm{a}}$ & $1,46^{a}$ & 0,062 \\
\hline Limon & \multicolumn{2}{|l|}{$4,67^{a}$} & $5,19^{b}$ & $5,54^{b}$ & $5,69 \mathrm{~b}$ & $<0,001$ \\
\hline Sable & \multicolumn{2}{|l|}{$94,14^{b}$} & $93,47^{a}$ & $93,32^{\mathrm{a}}$ & $92,85^{a}$ & $<0,001$ \\
\hline
\end{tabular}

Les moyennes affectées par la même lettre sur la même ligne ne sont pas statistiquement différentes au seuil de $5 \%$

II ressort du tableau 2 qu'il n'y a pas de différence significative entre les textures en fonction de la distance par rapport à la touffe. Selon les directions, une différence significative est observée avec une 


\section{Nomaou et al. J. Appl. Biosci. 2015 Effet des touffes de Guiera senegalensis (J.F. Gmel) sur la fertilité des sols dans la région de Maradi (Niger)}

teneur en argiles relativement plus importante du côté Est. Les variations en profondeur sont significatives au niveau des limons et des sables. On note en surface une diminution des limons compensée par les sables alors que les argiles restent statistiquement invariables. Tout semble croire que la touffe de Guiera senegalensis, même si elle joue un rôle dans le piégeage des sédiments transportés par le vent, ne retient qu'essentiellement que les sables (93\%). Aussi, il a été constaté que plus les touffes sont éloignées l'une de l'autre, moins elles captent les sédiments. L'introduction de la technique du marcottage dans la zone, pourrait aider à améliorer l'effet de Guiera senegalensis sur l'érosion, en permettant la formation d'un dôme entre les touffes.

$\mathrm{pH}$ : L'analyse de variance des valeurs de $\mathrm{pH}$ déterminées dans les sols est donnée dans le tableau 3.

Tableau 3: Analyse de variance des valeurs du pH des sols

\begin{tabular}{|c|c|c|c|c|c|c|}
\hline \multirow[t]{2}{*}{ Source de variance } & \multicolumn{5}{|c|}{ Les moyennes selon les distances } & \multirow[t]{2}{*}{ Fpr (5\%) } \\
\hline & $0 \mathrm{~cm}$ & $50 \mathrm{~cm}$ & $100 \mathrm{~cm}$ & $150 \mathrm{~cm}$ & $200 \mathrm{~cm}$ & \\
\hline $\mathrm{pH}$ & $5,69^{c}$ & $5,59^{b}$ & $5,51^{\mathrm{ab}}$ & $5,45^{\mathrm{a}}$ & $5,47^{\mathrm{a}}$ & $<0,001$ \\
\hline \multicolumn{7}{|c|}{ Les moyennes selon les directions } \\
\hline & E & W & & & $\mathbf{S}$ & Fpr (5\%) \\
\hline $\mathrm{pH}$ & $5,86^{c}$ & $5,53^{b}$ & & & $5,55^{b}$ & $<0,001$ \\
\hline \multicolumn{7}{|c|}{ Les moyennes selon les profondeurs } \\
\hline & $10 \mathrm{~cm}$ & $20 \mathrm{~cm}$ & & & $40 \mathrm{~cm}$ & Fpr $(5 \%)$ \\
\hline $\mathrm{pH}$ & $5,55^{\mathrm{a}}$ & $5,56^{\mathrm{a}}$ & & & $5,51^{\mathrm{a}}$ & 0,44 \\
\hline
\end{tabular}

Les moyennes affectées par la même lettre sur la même ligne ne sont pas statistiquement différentes au seuil de $5 \%$.

Le $\mathrm{pH}$ est acide quelque soit la modalité de comparaison considérée (distances, directions et profondeurs). Toutefois, il décroit de manière significative avec les distances. II est plus acide du côté Nord de la touffe et affiche des valeurs homogènes en profondeur. Cette acidité du sol sous Guiera senegalensis a été aussi mise en évidence par les travaux de Wezel et al. (2000) et Dossa et al. (2009) même si une légère amélioration dans les environs immédiats du Guiera senegalensis a été notée. On pourrait expliquer cette acidité par l'insuffisance d'apports des débris organiques sous Guiera senegalensis car il a été constaté qu'une bonne partie de ses feuilles qui tombent sont transportées par les vents d'Est - Ouest.

Matière organique: Les résultats d'analyse de variance des teneurs en carbone organique et en azote total sont consignés dans le tableau 4.

Tableau 4 : Analyse de variance des teneurs en carbone organique et en azote total des sols

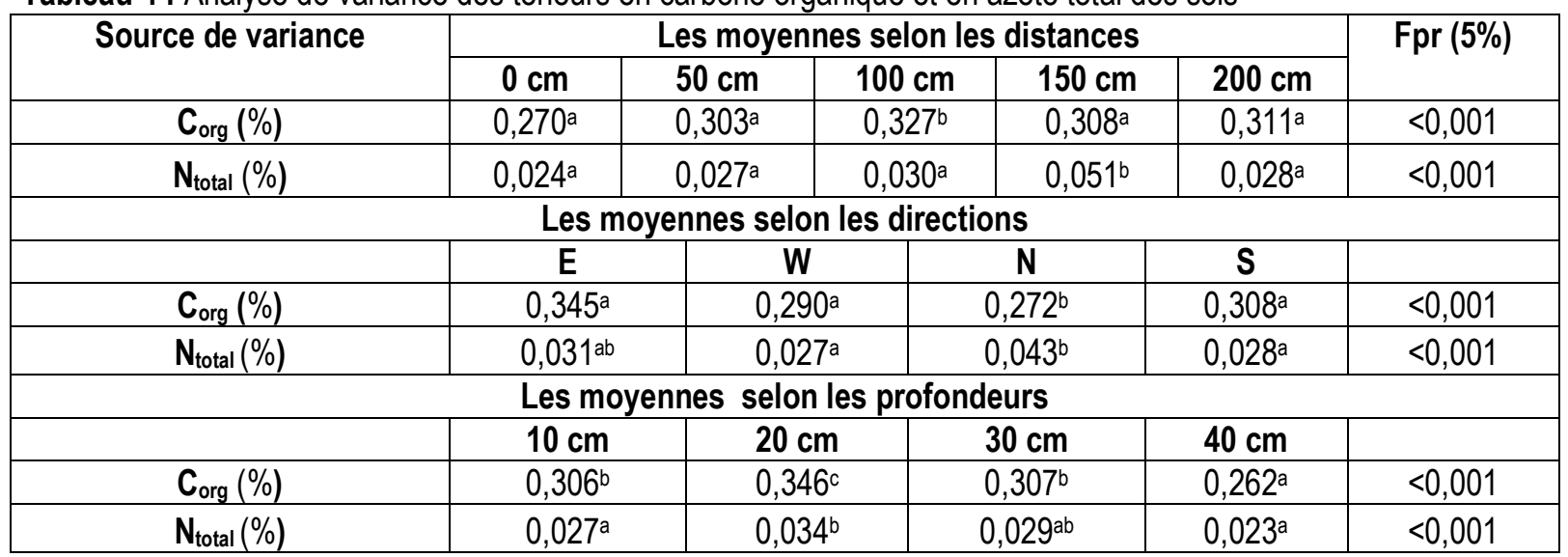

Les moyennes affectées par la même lettre sur la même ligne ne sont pas statistiquement différentes au seuil de $5 \%$ 


\section{Nomaou et al. J. Appl. Biosci. 2015 Effet des touffes de Guiera senegalensis (J.F. Gmel) sur la fertilité des sols dans la région de Maradi (Niger)}

L'analyse du tableau 4 permet de relever une différence significative du taux de carbone organique selon les distances et indique des valeurs proches $(0,3 \%)$ de celles des sols cultivés en mil (Evèquoz, 2000; Evèquoz et Guéro, 2000). En considérant les directions, on remarque que les côtés Est - Ouest Sud de la touffe ont des teneurs équivalentes. Seul le côté Nord affiche une teneur significative différente. La distribution de l'azote selon la distance indique des teneurs homogènes alors qu'elles sont beaucoup plus variables avec les directions. Ainsi, les valeurs les plus élevées sont relevées des côtés Nord et Est tandis que les directions Ouest et Sud affichent des valeurs équivalentes. En profondeur, on constate que les meilleurs taux d'azote sont enregistrés entre 20 et 30 $\mathrm{cm}$. Ces résultats sont comparables à ceux de Wezel et al. (2000) et Dossa et al. (2009). En outre, les taux de carbone organique et de l'azote total sensiblement acceptables trouvés autour des touffes de Guiera senegalensis, confirment le rôle d'amélioration de certains paramètres chimiques des sols joué par cette espèce comme l'ont relevé déjà certaines études (Larwanou et al., 2006 ; Lahmar et al., 2011 et Lahmar et Yacouba, 2012). Ce rôle est aussi souligné par Baumer (1987) qui disait que les arbres par leurs racines, exploitent le sol en profondeur et remontent les nutriments jusque dans leurs feuilles qui en tombant se décomposent à la surface du sol et les mettent à la disposition des cultures. Ces travaux montraient ainsi, qu'une parcelle accueillant 50 arbres/ha en alignement de plein champ stocke environ 1 tonne de carbone par ha/an dans le sol (sans compter la biomasse érigée). Cela démontre davantage le rôle des ligneux en général, dans la séquestration de carbone dans le sol.

Phosphores total et assimilable: Les résultats d'analyse statistique sont consignés dans le tableau 5 .

Tableau 5 : Analyse de variance des teneurs en phosphores total et assimilable des sols

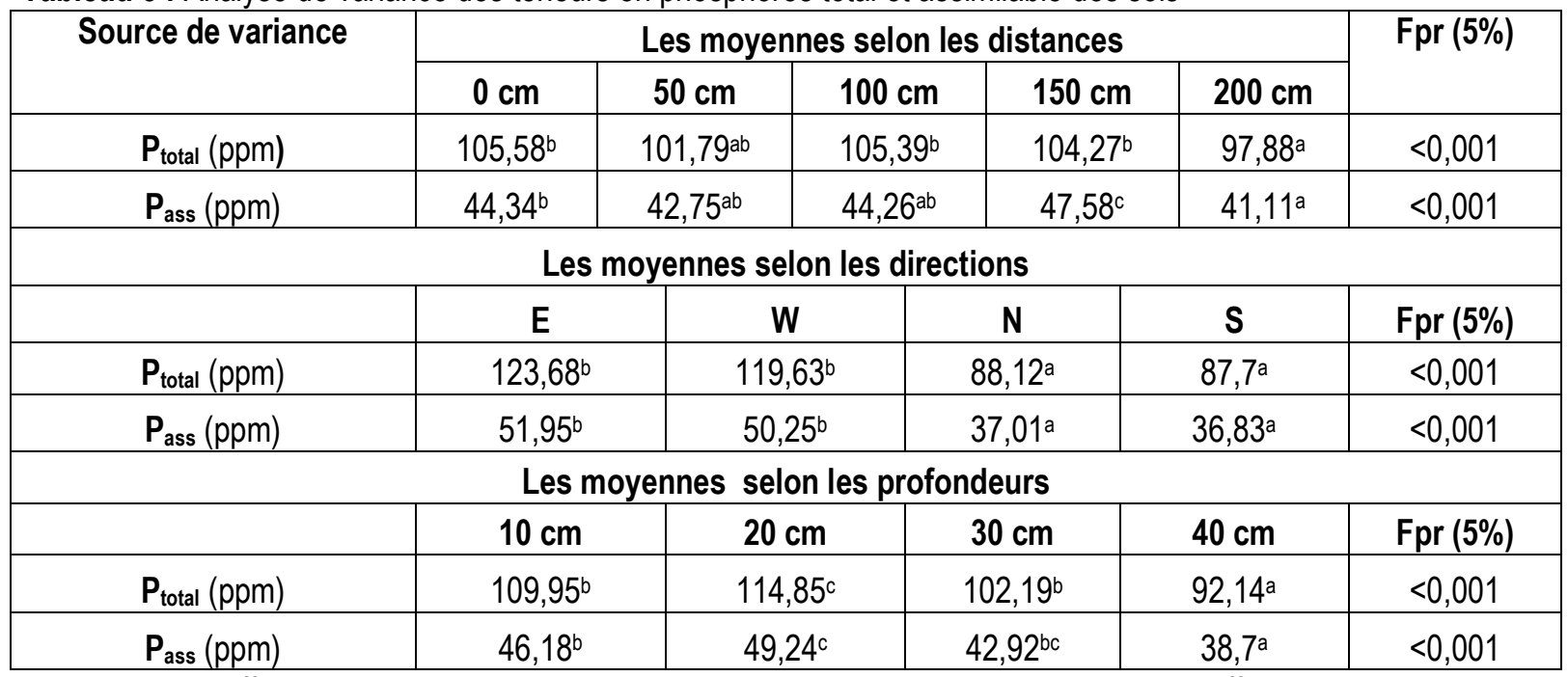

Les moyennes affectées par la même lettre sur la même ligne ne sont pas statistiquement différentes au seuil de $5 \%$

Les teneurs en phosphores total et assimilable affichent des différences significatives. Certes, elles diminuent à $50 \mathrm{~cm}$ de la touffe mais dans l'ensemble elles régressent lorsqu'on s'éloigne de celle - ci. Cette tendance régressive a été aussi relevée par Wezel et al., (2000) et Dossa al. (2009). Les directions Est Ouest enregistrent les meilleures teneurs comparativement aux deux autres directions. Les teneurs en phosphores décroissent avec la profondeur. Les bases échangeables: Compte tenu de très faibles valeurs du Na dans les sols (à l'état de traces), seules les trois principales bases $(\mathrm{Ca}, \mathrm{Mg}, \mathrm{K})$ ont été considérées. Le tableau 6 récapitule les teneurs moyennes de ces dernières en fonction de la distance à la touffe, la direction et la profondeur du sol. 


Nomaou et al. J. Appl. Biosci. 2015 Effet des touffes de Guiera senegalensis (J.F. Gmel) sur la
fertilité des sols dans la région de Maradi (Niger)

Tableau 6 : Analyse des variances des teneurs en bases échangeables des sols autour des touffes de Guiera senegalensis

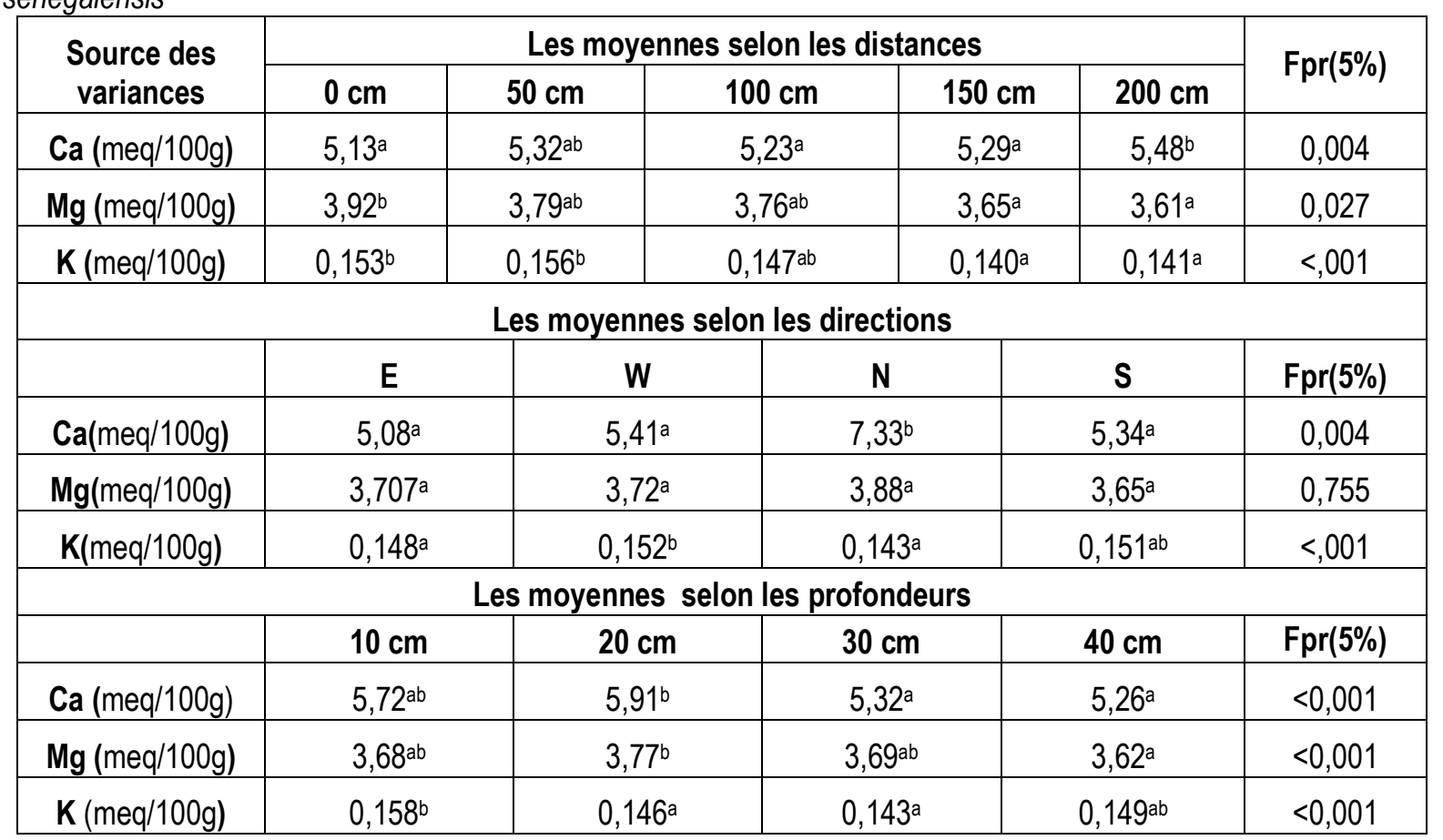

Les moyennes affectées par la même lettre sur la même ligne ne sont pas statistiquement différentes au seuil de $5 \%$

D'une manière générale, les teneurs en $\mathrm{Mg}$ et en $\mathrm{K}$ diminuent significativement lorsqu'on s'éloigne de la touffe de Guiera senegalensis contrairement à celles du Ca qui augmentent avec la distance ce qui corrobore les résultats de Wezel et al. (2000). Les teneurs moyennes en $\mathrm{Ca}$ et $\mathrm{K}$ varient significativement selon les directions avec respectivement 5,08 à 7,33 et 0,143 à $0,158 \mathrm{meq} / 100 \mathrm{~g}$, à l'inverse $\mathrm{du} \mathrm{Mg}$ dont les teneurs sont statistiquement peu variables $(3,65-3,88$ $\mathrm{meq} / 100 \mathrm{~g}$ ) quelque soit la direction. Le profil des 3 bases indique globalement une diminution significative des teneurs moyennes avec la profondeur. Ces différentes variations des principales bases échangeables peuvent être liées aux apports des sédiments éoliens et aux substrats organiques captés par les touffes de Guiera senegalensis et probablement aux éléments minéraux des horizons profonds que les racines de Guiera senegalensis ont pu remonter en surface (Louppe, 1991).

La capacité d'échange cationique (CEC): Les résultats d'analyse de variance de la CEC dans les sols sont donnés dans le tableau 7.

Tableau 7 : Analyse de variance des valeurs de la CEC des sols

\begin{tabular}{|c|c|c|c|c|c|c|c|}
\hline \multirow[t]{2}{*}{ Source de variance } & \multicolumn{6}{|c|}{ Les moyennes selon les distances } & \multirow[t]{2}{*}{ Fpr $(5 \%)$} \\
\hline & $0 \mathrm{~cm}$ & $50 \mathrm{~cm}$ & & $00 \mathrm{~cm}$ & $150 \mathrm{~cm}$ & $200 \mathrm{~cm}$ & \\
\hline CEC (meg/100g) & $10,52^{\mathrm{ab}}$ & $10,57^{b}$ & & $0,55^{b}$ & $10,50^{\mathrm{a}}$ & $10,62^{b}$ & 0,021 \\
\hline \multicolumn{8}{|c|}{ Les moyennes selon les directions } \\
\hline & $\mathrm{E}$ & & & & & $\mathbf{S}$ & Fpr $(5 \%)$ \\
\hline CEC (meg/100g) & $10,42^{a}$ & & & & & $10,57^{b c}$ & $<0,01$ \\
\hline \multicolumn{8}{|c|}{ Les moyennes selon les profondeurs } \\
\hline & $10 \mathrm{~cm}$ & & & & & $40 \mathrm{~cm}$ & Fpr (5\%) \\
\hline CEC (meq/100g) & $10,52^{\mathrm{a}}$ & & & & $57^{a}$ & $10,51^{a}$ & 0,85 \\
\hline
\end{tabular}

Les moyennes affectées par la même lettre sur la même ligne ne sont pas statistiquement différentes au seuil de $5 \%$ 


\section{Nomaou et al. J. Appl. Biosci. 2015 Effet des touffes de Guiera senegalensis (J.F. Gmel) sur la fertilité des sols dans la région de Maradi (Niger)}

Les valeurs moyennes de la CEC varient significativement de 10,50 à $10,62 \mathrm{meq} / 100 \mathrm{~g}$ et de 10,42 à 10,60 meq/100 g respectivement pour les distances et les directions. Pour les profondeurs, elles sont homogènes $(10,51$ à 10,57 meq/100 g). Ces

\section{CONCLUSION}

L'étude des touffes de Guiera senegalensis dans le terroir de Guidan Bakoye a permis de relever une très forte densité de cette espèce qui avait disparu il y a une trentaine d'années. Cette forte régénération de Guiera Senegalensis dans cette localité est principalement favorisée d'une part, par l'adoption de la technique de régénération naturelle assistée et d'autre part, par la bonne organisation des paysans de Guidan Bakoye en comité de surveillance. Par la suite, l'introduction du marcottage vient renforcer davantage le développement de cette espèce dans le terroir car un taux de réussite de $90 \%$ a été obtenu. L'analyse des résultats obtenus sur l'influence des touffes de Guiera senegalensis sur certains paramètres physicochimiques des sols de terroir de Guidan Bakoye fait ressortir l'absence de changement textural avec la distance et la prédominance des sables dans les horizons de surface démontrant ainsi que les touffes de Guiera senegalensis interceptent essentiellement les

\section{RÉFÉRENCES BIBLIOGRAPHIQUES}

Abdou M.M., Alzouma Mayaki Z., Kadri A., Ambouta J.M.K. et Dan Lamso N., 2013. Effet de l'arbre Acacia senegal sur la fertilité des sols de gommeraies du Niger. Int. J. Biol. Chemi. Sci., 7 (6) : 2328 - 2337.

Adam T., Reij C., Abdoulaye T., Larwanou M., Tappan G. et Yamba B., 2006. Impacts des investissements dans la gestion des ressources naturelles (GRB) au Niger: Rapport de synthèse. Étude sahélienne, Centre Régional d'Enseignement Spécialisé en Agriculture (CRESA), Niamey - Niger. $54 \mathrm{p}$.

Ambouta J.M.K., Issaka A. et Issa S.1998. Gestion de la fertilité des sols et évolution des sols de Gakudi (Maradi, Niger). Cahiers Agricultures $7: 395$ - 400 .

Arbonnier M., 2009. Arbres, arbustes et lianes des zones sèches d'Afrique de l'Ouest. $3 \mathrm{e}$ ed. Versailles, QUAE, $572 \mathrm{p}$.

Baize D., 1988. Guide des analyses courantes en pédologie, choix, expression, présentation, interprétation. INRA, Paris, $172 \mathrm{p}$. valeurs moyennes de la CEC pourraient s'expliquer probablement par l'absence constatée de l'augmentation des teneurs en argile autour des touffes de Guiera senegalensis et aussi par l'apport insuffisant de matière organique.

éléments grossiers transportés par les vents. Sur le plan chimique, les résultats obtenus montrent que les sols sous touffes de Guiera senegalensis présentent un $\mathrm{pH}$ acide quelque soit la variable considérée (distance, direction, profondeur), indiquant que la présence de l'arbuste Guiera senegalensis ne semble pas améliorer le $\mathrm{pH}$ du sol. Concernant la matière organique et le phosphore, il est noté une nette diminution de leurs teneurs avec la distance et la profondeur, qui serait liée à l'interception des débris végétaux par les touffes de Guiera senegalensis. S'agissant des bases échangeables, il est relevé une influence de Guiera senegalensis sur les teneurs en $\mathrm{Mg}$ et $\mathrm{K}$ qui diminuent avec la distance contrairement au $\mathrm{Ca}$. La capacité d'échanges cationiques quant à elle, ne change pas quelque soit la modalité considérée. En somme, les touffes de Guiera senegalensis améliorent moyennement les propriétés physico-chimiques du sol.

Bationo B.A., 1994. Étude des potentialités agroforestières, de la multiplication et des usages de Guiera senegalensis J.F.GMEL. Mémoire de fin d'études. Institut de Développement Rural / Université de Ouagadougou. $73 \mathrm{p}$.

Bationo B.A., Karim S., Bellefontaine R., Saadou M. Guinko S., Ichaou A., Bouhari A., 2005. Le marcottage terrestre : technique économique pour la régénération de certains ligneux tropicaux. Sécheresse 16 : 309-311.

Bationo B.A., Kalinganiré A. et Bayala J., 2012. Potentialités des ligneux dans la pratique de l'agriculture de conservation dans les zones arides et semi arides de l'Afrique de l'Ouest : Aperçu de quelques systèmes candidats. ICRAF Technical Manual no. 17 Nairobi : World Agroforestry Centre, 32P.

Baumer M., 1987. Le rôle de l'agroforesterie dans la lutte contre la désertification et la dégradation de l'environnement, ICRAF / CTA : Washington. $260 \mathrm{p}$. 


\section{Nomaou et al. J. Appl. Biosci. 2015 Effet des touffes de Guiera senegalensis (J.F. Gmel) sur la fertilité des sols dans la région de Maradi (Niger)}

Dan Lamso N, Guero Y., Tankari Dan-Badjo A., Rabah L., Andre B. B., Patrice D., Tidjani A. D., Ado Maman N., Ambouta Jean Marie K., 2015. Variations texturales et chimiques autour des touffes d'hyphaene thebaica (mart) des sols dans la region de maradi (niger). Algerian journal of arid environment, 5 (1): 40-55

Dossa E.L., Khouma M., Diedhiou I., Sene M., Kizito F., Badiane A.N., Samba S.A.N., Dick R.P., 2009. Carbon, Nitrogen and phosphorus mineralization potential of semi-arid Sahelian soils amended with native shrub residues. Geoderma, 148: 251-260.

Evèquoz M., 2000. Dynamique de la matière organique et durabilité des systèmes de production nord sahéliens. Annales de l'Université Abdou MOUMOUNI de Niamey. Numéro hors série : $11-28$.

Evequoz M., Guero Y., 2000. Conservation et Gestion des eaux et des sols: Durabilité des systèmes de production nord sahélien. Programme de Conservation et Gestion des Eaux et des Sols au Niger. Univ. Abdou Moumouni (Niamey) and Ecole Polytechnique de Zurich, $120 \mathrm{p}$.

Hamissou A., 2005. Étude de faisabilité technique, économique et organisationnelle d'un marché à bois issu de la RN dans la grappe de Dan Saga. Mémoire CRESA, Faculté d'Agronomie / Université Abdou Moumouni de Niamey, 71 p.

Kadadé A., 1999. Système de production et gestion de la fertilité des sols dans la rôneraie de Gaya: cas de terroir de Bana. Mémoire de fin d'étude/CRESA /Niamey. 68 pages.

Lahmar, R., Bationo, B.A., Dan Lamso, N., Guéro, Y., Tittonell, P., 2011. Tailoring conservation agriculture technologies to West Africa semiarid zones: building on traditional local practices for soil restoration. Field Crop Res., doi: 10.1016/j.fcr.2011.09.013.

Lahmar R., Yacouba H., 2012. Zaï et potentiel de l'association cultures annuelles-arbustes natifs. In: Dia Abdoulaye (ed.), Duponnois Robin (ed.). La grande muraille verte : capitalisation des recherches et valorisation des savoirs locaux. Marseille : IRD [Marseille], p. 203-223.

Larwanou M., Saadou M. et Hamadou S., 2006. Les arbres dans les systèmes agraires en zone sahélienne du Niger : mode de gestion, atouts et contraintes. Tropicultura, 24 (1) : $14-18$.
Louppe D., 1991. Guiera senegalensis, espèce agroforestière? Micro-jachère dérobée de saison sèche et approvisionnement énergétique d'un village du centre nord du Bassin Arachidier sénégalais. Bois et Forêts des Tropiques, $228:$ 41-47.

Mai Moussa K.A., 1996. Environnement de Faidherbia Albida Del; caractérisation; exploitation et perspectives d'optimisation dans les zones soudano-sahéliennes de l'Afrique de l'Ouest. Thèse de $3^{\text {ème }}$ cycle pour le doctorat université de Cocody / Cote d'lvoire $137 \mathrm{p}$.

Maï Moussa K.A, Williams J.H. \& Odongo J.C.W., 1997. Diversification des cultures sous Faidherbia albida en milieu paysan dans la zone semiaride de l'Afrique de l'ouest. In: $\mathrm{G}$. Renard, A. Neef, K. Beckert and M. von Oppen (eds.): Soil fertility management in west African land use systems. Proceedings of the regional workshop. Margraf verlag. Niamey, Niger 4-9 March 1997. p. 299-303.

Moussa H., 1997. Germination du palmier doum (Hyphaene thebaica Mart.) et analyse de son interaction avec le mil ( Pennisetum glaucum $L, R, B r$.) en zone semi-aride du Niger, Thèse de Philosophie doctorale (PhD) de Université Laval/ Canada, $178 \mathrm{p}$.

Moustapha A., 2005. Étude de faisabilité technique, économique et organisationnelle d'un marché à bois issu de la RN dans la grappe de Dan Saga. Mémoire ITA, Faculté d'Agronomie / Université Abdou Moumouni de Niamey, Niger $67 \mathrm{p}$.

Salissou A., 2004. Valorisation des produits et sous produits ligneux dans la partie Nord $d u$ département d'Aguié : cas du terroir villageois de Dan Saga. Mémoire ITA, Faculté d'Agronomie / Université Abdou Moumouni de Niamey, Niger, $66 \mathrm{p}$.

Strebelle J. and Boubacar B., 2011. Sécurité alimentaire et organisations intermédiaires: évaluation et identification des besoins de renforcement des capacités des organisations paysannes dans six pays de l'UEMOA et de la CEDEAO. Participation des organisations paysannes et leurs faîtières à la sécurité alimentaire et aux flux commerciaux dans les marchés des produits de base. Rapport pays : Niger. Projet WAF/6349. Collectif Stratégies Alimentaires, Bruxelles - Belgique, 64p. 
Nomaou et al. J. Appl. Biosci. 2015 Effet des touffes de Guiera senegalensis (J.F. Gmel) sur la fertilité des sols dans la région de Maradi (Niger)

Thiombiano A., Schmidt M., Kreft H., Guinko S., 2006. Influence du gradient climatique sur la

Wezel A., Rajot- J.L., Herbrig C., 2000. Influence of shrubs on soil characteristics and their function distribution des espèces de Combretaceae au Burkina Faso (Afrique de l'Ouest). Candollea, in Sahelian agro-ecosystems in semi-arid Niger. Journ. Arid Environ, 44: 383-398. $61: 189-213$. 